\title{
REDUÇÃO DE PERDAS EM REDES DE DISTRIBUIÇÃO DE ENERGIA ELÉTRICA ATRAVÉS DE SISTEMAS CLASSIFICADORES
}

\author{
Patrícia Amâncio Vargas ${ }^{\dagger}$ \\ pvargas@dca.fee.unicamp.br
}

\author{
Christiano Lyra Filho* \\ chrlyra@densis.fee.unicamp.br
}

\section{Fernando José Von Zuben ${ }^{\dagger}$ \\ vonzuben@dca.fee.unicamp.br}

${ }^{*}$ Depto. de Engenharia de Sistemas, Faculdade de Engenharia Elétrica e de Computação, Universidade Estadual de Campinas, C. P. 6101, 13081-970, Campinas, SP - Brasil

${ }^{\dagger}$ Depto. de Engenharia de Computação e Automação Industrial, Faculdade de Engenharia Elétrica e de Computação, Universidade Estadual de Campinas, C. P. 6101, 13081-970, Campinas, SP - Brasil

\begin{abstract}
The problem of minimising the technical energy losses in electric power distribution systems corresponds to the definition of the best network configuration, through the manipulation of sectionalising switches. Using a graph terminology, we have a combinatorial optimisation problem, whose solution asks for the definition of the minimum spanning tree for the network. With the purpose of treating the case of time-varying profiles of energy requirements, this paper presents the first results obtained with the application of learning classifier systems, an evolutionary computation approach to find network configurations close to the one associated with minimum energy losses. Its uttermost feature is the possibility of application to on-line network supervision, proposing new configurations that will reduce the level of energy losses when significant demand variations are detected.
\end{abstract}

KEYWORDS: Power distribution, loss minimisation, time-varying demand, learning classifier systems, evolutionary computation.

\section{RESUMO}

O problema de minimização de perdas técnicas em redes de distribuição de energia elétrica corresponde à definição da melhor configuração da rede, através do controle do estado das chaves seccionadoras do sistema. Usando uma terminologia de grafos, resulta um problema de otimização combinatória cuja solução requer a definição de uma árvore geradora de custo mínimo para a rede. Com o propósito de tratar o caso de perfis de demanda variantes no tempo, este trabalho apresenta os primeiros resultados obtidos com o uso do conceito de sistemas classificadores, uma abordagem de computação evolutiva, para encontrar configurações de rede próximas às de perdas mínimas. Seu principal atrativo é a possibilidade de aplicação à supervisão de redes em tempo real, propondo configurações que levem à redução de perdas sempre que forem identificadas variações significativas de demanda.

PALAVRAS-CHAVE: Distribuição de energia elétrica, minimização de perdas, demanda variante no tempo, sistemas classificadores, computação evolutiva.

Artigo submetido em $20 / 12 / 00$

1a. Revisão em 09/06/03

Aceito sob recomendação do Ed. Assoc. Prof. José L. R. Pereira 


\section{INTRODUÇÃO}

Este trabalho explora a utilização da computação evolutiva, em particular de sistemas classificadores (Holland, 1992), como alternativa de solução do problema de redução de perdas em sistemas de distribuição de energia elétrica.

Baseado nas características do problema, os sistemas classificadores definem, a partir de um processo computacional de treinamento evolutivo, um conjunto de regras, chamadas de classificadores. Para definição do conjunto de classificadores ótimos, interpretado como uma população, aplicam-se os procedimentos intrínsecos à computação evolutiva: codificação genética, seleção, recombinação e mutação.

Usando uma terminologia de grafos, o problema de minimização de perdas na distribuição de energia elétrica corresponde à definição de uma árvore geradora de custo mínimo para a rede. No entanto, quando os pesos nos arcos, diretamente vinculados às perdas, variam com os fluxos, o problema não pode ser resolvido com os algoritmos muito eficientes, do tipo "guloso", já propostos na literatura. De fato, a busca de soluções ótimas para grafos com pesos variáveis é um problema de grande complexidade computacional. Isto motivou o desenvolvimento de métodos heurísticos para abordar o problema de minimização de perdas em redes de distribuição, como "abertura seqüencial de chaves" (Merlin \& Back, 1975; Shirmohammadi \& Hong, 1989; Peponis et al., 1995) e "troca de ramos" (Civanlar et al., 1988; Baran \& Wu, 1989; Peponis et al., 1995).

Merlin \& Back (1975), pioneiros na proposição e abordagem do problema, já identificaram dificuldades para encontrar uma configuração de perdas mínimas através de algoritmos exatos. De fato, as abordagens mais utilizadas conduzem a soluções aproximadas. Cavellucci \& Lyra (1997) ampliaram a possibilidade de encontrar a solução de mínimas perdas, mas a abordagem ainda tem limitações para utilização em redes de grande porte.

Nara et al. (1992) foram os primeiros autores a investigar o uso de computação evolutiva para redução de perdas em sistemas de distribuição de energia elétrica. A estratégia desenvolvida por Nara e co-autores levava à construção de soluções que violavam a restrição de radialidade. O processo de identificação e eliminação das soluções infactíveis comprometia o desempenho da abordagem. Costa (1999) resolveu este problema ao acrescentar inovações metodológicas de computação evolutiva e explorar a possibilidade de usar estes conceitos juntamente com busca tabu (Glover \& Laguna, 1997).
As abordagens descritas acima supõem que as demandas de energia são fixas. Este trabalho, por sua vez, procura estender os resultados de Costa (1999) para o tratamento de redes com variação das demandas nos nós (Vargas et al., 2001), ou seja, centros consumidores de energia. Para tanto, recorre-se aos sistemas classificadores, os quais apresentam grande potencial de adaptação a "ambientes" variantes no tempo. Outra contribuição do trabalho é propor uma codificação genética em ponto flutuante, em lugar da codificação binária comumente utilizada na abordagem de problemas de minimização de perdas.

Na próxima seção, apresentamos o problema de minimização de perdas técnicas em sistemas de distribuição de energia elétrica, suas formas de representação e formulação matemática.

Na Seção 3, expomos os conceitos básicos de algoritmos genéticos. Em seguida, mostramos os detalhes da aplicação dessa ferramenta ao problema de minimização de perdas em sistemas de distribuição de energia elétrica, juntamente com resultados e comentários.

Os conceitos de Sistemas Classificadores são apresentados na Seção 4, onde são detalhados os subsistemas que o compõem.

Na Seção 5, apresentamos as particularidades da aplicação dos Sistemas Classificadores ao problema de minimização de perdas, juntamente com resultados e comentários.

Seguem-se conclusões, agradecimentos e referência bibliográfica.

\section{FORMULAÇÃO E MOTIVAÇÃO DO PROBLEMA}

As perdas técnicas em redes de distribuição de energia elétrica podem ser reduzidas através da substituição de linhas, usando-se materiais de menor resistência, da instalação de capacitores e de reconfigurações.

As reconfigurações de redes são as que apresentam as implantações mais econômicas, seguidas da utilização de capacitores. Mantendo-se a estrutura radial, as reconfigurações são realizadas por abertura e fechamento de chaves seccionadoras do sistema.

O problema de obter a configuração de rede com menor perda de energia pode ser formulado como segue: 


$$
\operatorname{Min} \sum_{k=0}^{n-1} r_{k} \frac{P_{k}^{2}+Q_{k}^{2}}{V_{k}^{2}}
$$

s.a: Suprimento de Demanda

Limites de Fluxo

Operação Radial

onde $r_{k}$ é a resistência da linha, $P_{k}$ é o fluxo de potência ativa, $Q_{k}$ é o fluxo de potência reativa $\mathrm{e} V_{k}$ é a tensão na barra $k$. $P_{k}, Q_{k}$ e $V_{k}$ são calculados por equações recursivas de fluxo de carga, as quais são estruturalmente ricas e propícias a esquemas de solução computacionalmente eficientes (Baran \& Wu, 1989).

Como conseqüência da restrição de radialidade, a solução do problema corresponde à definição de uma árvore geradora de custo mínimo para a rede (Ahuja et al., 1993). No entanto, como os pesos nos arcos (as perdas) variam com o nível de demanda em cada nó do grafo, o problema não pode ser resolvido com algoritmos que supõem que os arcos têm pesos fixos. Trata-se de um problema de otimização combinatória e com variação de parâmetros ao longo do tempo, em que o esforço computacional para se encontrar a solução ótima por avaliações exaustivas envolveria a análise de todas as árvores geradoras possíveis (Baran \& Wu, 1989). Esta busca exaustiva é computacionalmente impraticável, principalmente no caso de nós com demandas variáveis.

A viabilidade do trato computacional é conquistada à medida que se abre mão da garantia de obtenção da solução ótima em prol de uma boa solução.

Mas, mesmo entre os algoritmos aproximados, a maior parte supõe que o perfil de demandas é fixo. Dentre estes, se encontra uma versão de algoritmo genético que será empregada neste trabalho para demonstrar que as soluções ótimas (estados das chaves seccionadoras) sofrem modificações significativas para diferentes perfis de demanda. Neste caso, estarão sendo abordadas várias situações distintas com perfis de demanda fixos.

Uma vez demonstrado que existe uma configuração ótima de chaves que é diferente para cada perfil de demanda, então se justifica a supervisão em tempo real, capaz de indicar novas reconfigurações para a rede de distribuição de acordo com a evolução do perfil de demanda e visando manter a rede sempre o mais próximo possível de uma situação de perdas mínimas.

Apresentamos a seguir conceitos básicos e os detalhes da aplicação de algoritmos genéticos na busca da redução de perdas em sistemas de distribuição de energia elétrica.

\section{ENTRADA : POPULAÇÃO INICIAL FUNÇÃO DE FITNESS}

REPITA: INÍCIO DO PROCESSO EVOLUTIVO (GERAÇÕES)

Passo 1 : Seleção:

- Selecionar indivíduos (pais) de acordo com o fitness. Passo 2: Reprodução :

- Aplicar recombinção (a um par)

- Aplicar mutação

Passo 3 : Formar uma nova população com os filhos gerados

Passo 4 : Calcular novo Fitness de cada indivíduo da população

ATÉ QUE O CRITÉRIO DE PARADA SEJA

ATENDIDO

\section{SAÍDA: MELHOR INDIVÍDUO PRESENTE NA ÚLTIMA GERAÇÃO}

Figura 1: Passos básicos do algoritmo genético

\section{ALGORITMOS GENÉTICOS}

Os algoritmos ditos genéticos são caracterizados por um conjunto predeterminado e bem definido de regras e processos com operações finitas, destinados à busca estocástica polarizada da solução de um problema (Holland, 1992). São implementados com inspiração nas leis biológicas da transmissão dos caracteres hereditários entre as gerações de uma dada espécie, empregando operadores genéticos de recombinação e mutação.

Na figura 1, apresentamos de forma simplificada os passos básicos do processo de execução de um algoritmo genético. Ele está representado aqui como uma função que recebe como entrada uma população inicial e uma função de avaliação, normalmente denominada fitness, e fornece como saída o melhor indivíduo encontrado após o processo evolutivo.

Observa-se que o algoritmo genético parte de uma população inicial. Através de um mecanismo de seleção, dá preferência aos indivíduos mais adaptados ao ambiente (que apresentam maior fitness) e aplica os operadores genéticos de recombinação ('crossover') e mutação.

Caracteriza-se assim um processo evolutivo, em que se espera que o fitness do melhor indivíduo a cada geração apresente uma tendência de crescimento continuado, sendo este indivíduo, por conseguinte, capaz de representar uma boa solução para o problema proposto (a qual pode não ser a melhor possível). 
Cada indivíduo é considerado um "cromossomo" e, na representação clássica de algoritmos genéticos, ele é codificado como um vetor de tamanho fixo, formado pela concatenação dos caracteres " 0 " e "1" do alfabeto binário $\{0,1\}$ (representação binária). No entanto, existem variações para esta codificação, como a representação em ponto flutuante, onde o vetor de tamanho fixo é formado pela concatenação de números reais (Michalewicz, 1996). Esta possibilidade foi explorada em nossa implementação para o problema de minimização de perdas, relatado a seguir.

\subsection{Algoritmos Genéticos Aplicados ao Problema de Minimização de Perdas}

Conforme exposto na Seção 2, o problema de minimização de perdas em sistemas de distribuição de energia elétrica, caracteriza uma situação de otimização combinatória.

Este problema foi abordado por Costa (1999) a partir da aplicação de algoritmos genéticos com representação binária, juntamente com inovações na representação genética de estruturas em árvore. Com o objetivo de confirmar a necessidade de se encontrar soluções distintas (estado das chaves seccionadoras) para redução de perdas em cada perfil diferente de demanda, incorporamos representação genética em ponto flutuante ao algoritmo de solução para situações de demandas fixas proposto por Costa (1999).

A representação em ponto flutuante mostrou-se mais adequada, por preservar a noção de vizinhança na aplicação do operador de mutação, o que não ocorre com a representação binária.

Para cada vetor de tamanho fixo (indivíduo), onde cada número representa o peso fictício de um arco da rede, sempre vai existir uma árvore de custo mínimo associada (Ahuja et al., 1993). Portanto, em lugar de se atuar diretamente na evolução do estado das chaves seccionadoras, pode-se evoluir apenas pesos fictícios atribuídos aos arcos no grafo. Desta forma, após a aplicação dos operadores genéticos intrínsecos ao processo evolutivo, estes pesos fictícios são utilizados como argumentos de entrada para um algoritmo que obtém uma árvore de custo mínimo.

Ao se evitar a busca da árvore de mínimas perdas por atuação direta na determinação do estado das chaves seccionadoras, optando-se pela busca de pesos fictícios para o grafo, elimina-se a possibilidade de existência de soluções infactíveis, originadas da existência de restrições a serem atendidas (observe a formulação do pro- blema em (1)). O tratamento direto do estado das chaves a partir de uma codificação binária $(0=$ chave aberta, 1 = chave fechada) levaria a soluções candidatas, ou seja, configurações de chaves, que violariam as restrições do problema.

Sendo assim, busca-se um conjunto de pesos fictícios para o grafo, sendo que a cada conjunto de pesos fictícios sempre existe uma árvore de custo fictício mínimo associada, a qual representa sempre uma configuração factível para a rede. Logo, o objetivo é obter uma árvore de custo fictício mínimo (custo este relativo apenas aos pesos fictícios vinculados à codificação genética) que também represente a árvore de perdas mínimas (as perdas aqui são relativas ao estado real das perdas na rede de distribuição).

Justifica-se a codificação em ponto flutuante pelo fato dos pesos fictícios dos arcos estarem definidos no intervalo $[0,1]$. A árvore de custo mínimo apontará quais chaves seccionadoras (do conjunto total de chaves da rede) devem ficar abertas. Repare que o perfil de demanda real apresentado pelo sistema de distribuição só será utilizado para avaliar a qualidade da árvore proposta, definindo assim o seu valor de fitness.

Teremos um vetor com tantos pesos fictícios em ponto flutuante quantos forem os arcos do sistema. A decodificação do vetor de pesos fictícios (cromossomo) segue a metodologia apresentada em Costa (1999). Costa utilizou o algoritmo de Prim para gerar estruturas do tipo árvore com custo mínimo (condição necessária para o processo de evolução da população), a partir de um conjunto de arcos com peso fixo. Em nosso caso, adotamos o algoritmo de Sollin, mais indicado para a geração de árvores esparsas (Ahuja et al., 1993) - situação típica das redes de distribuição de energia elétrica.

Em todos os casos a serem considerados nesse trabalho, será utilizada a rede de distribuição de energia elétrica ilustrada na figura 2, a qual foi proposta inicialmente por Baran \& Wu (1989). Ela é composta por 37 arcos (supomos a existência de uma chave seccionadora para cada arco) e 32 nós. Na configuração apresentada, 5 chaves seccionadoras estão abertas: 33, 34, 35, 36 e 37.

Para a rede apresentada na figura 2, selecionamos seis perfis com propostas de distribuições aleatórias de demandas entre os nós da rede. Estes perfis são distintos, mas a soma das demandas de energia (demanda total) foi mantida constante.

Para um dado perfil de demandas nos nós, buscamos minimizar as perdas de energia elétrica da rede através do emprego de algoritmos genéticos. 


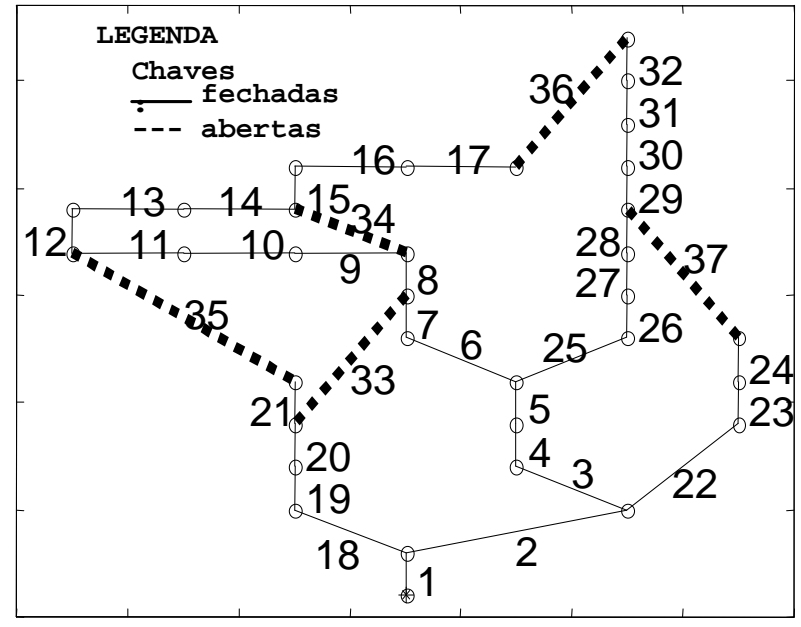

Figura 2: Rede de Distribuição (Baran \& Wu, 1989)

Tabela 1: Perfis de Consumo $\times$ Configurações

\begin{tabular}{|c|c|c|c|c|c|c|}
\hline & $\mathrm{P}_{1}$ & $\mathrm{P}_{2}$ & $\mathrm{P}_{3}$ & $\mathrm{P}_{4}$ & $\mathrm{P}_{5}$ & $\mathrm{P}_{6}$ \\
\hline $\mathrm{P}_{1}$ & 0 & 0,2911 & 2,2553 & 0,3059 & 1,7839 & 6,4485 \\
\hline $\mathrm{P}_{2}$ & 1,7181 & 0 & 1,0184 & 12,602 & 0,4144 & 4,2648 \\
\hline $\mathrm{P}_{3}$ & 1,5189 & 2,5179 & 0 & 13,277 & 0,1080 & 4,1104 \\
\hline $\mathrm{P}_{4}$ & 2,7524 & 2,8187 & 12,481 & 0 & 9,4363 & 12,819 \\
\hline $\mathrm{P}_{5}$ & 3,8348 & 2,4171 & 3,9084 & 16,925 & 0 & 1,5433 \\
\hline $\mathrm{P}_{6}$ & 17,918 & 32,532 & 21,187 & 26,369 & 17,757 & 0 \\
\hline
\end{tabular}

Como resultado desse estudo, identificou-se a necessidade de se definir configurações de rede distintas para cada perfil de demanda.

A tabela 1 ilustra o aumento das perdas, em percentagem, de uma determinada configuração "ótima", encontrada para um perfil de demanda $\mathbf{P}_{i}(i=1, \ldots, 6)$, quando aplicada a outro perfil $\mathbf{P}_{j}(j=1, \ldots, 6, j \neq i)$. Por exemplo, a utilização no caso do perfil $\mathbf{P}_{6}$ da melhor configuração encontrada para o perfil $\mathbf{P}_{1}$ (chaves abertas: 7,10 , 14, 16 e 28), resultou em um acréscimo de $6,4485 \%$ nas perdas, em relação à melhor configuração encontrada para $\mathbf{P}_{6}$ (chaves abertas: 7, 9, 14, 32 e 37 ).

Para lidar com a situação de perfil de demanda variável, recorremos a uma outra ferramenta da Computação Evolutiva, os Sistemas Classificadores. A principal motivação para a utilização de sistemas classificadores é seu potencial de adaptação a "ambientes" variantes no tempo e que requerem ações contínuas, muitas vezes em tempo real (Booker et al., 1989).

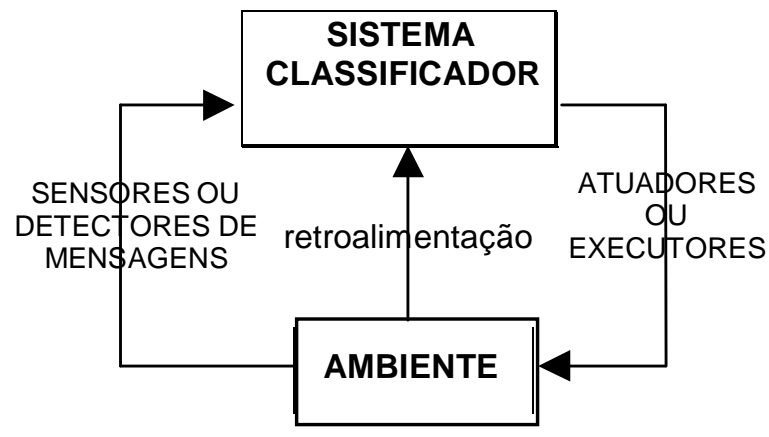

Figura 3: Interação entre o Sistema Classificador e o Ambiente

\section{SISTEMAS CLASSIFICADORES}

O conceito de Sistemas Classificadores foi proposto originalmente por Holland em meados dos anos 70 (Holland, 1992) - na literatura em língua inglesa os sistemas classificadores são denominados "Learning Classifier Systems" (LCS). É uma das abordagens que se inspiram em processos da natureza para solução de problemas. Basicamente, consiste em uma metodologia para criação e atualização de regras (os classificadores), que codificam alternativas de ações específicas para as características do problema (Booker et al., 1989).

Os sistemas classificadores vem sendo aplicados com sucesso a problemas do mundo real. Dentre estes se destacam as áreas de "data mining", comércio eletrônico, simulações de aeronaves, roteamento de redes de telecomunicação, robótica móvel, controle de tráfego, controle de operação de gás natural encanado, otimização de formas geométricas obedecendo a critérios de qualidade em instrumentos industriais e modelagem de mercado para fornecedores de energia elétrica (Lanzi et al., 2000; Lanzi et al., 2001).

Esta seção descreve os principais aspectos da arquitetura de sistemas classificadores proposta por Holland, adotada no trabalho. Observa-se, no entanto, que existem arquiteturas alternativas (Vargas et al., 2003).

A figura 3 ilustra em linhas gerais o processo de interação com o ambiente de um sistema classificador. A comunicação entre o sistema classificador e o ambiente sobre o qual ele atua ocorre através de seus detectores de mensagens e de seus executores. Os detectores são responsáveis pela recepção e codificação da(s) mensagem(ns) recebida(s), transformando-a(s) em uma linguagem inteligível ao sistema classificador. O sistema age sobre o ambiente através de seus atuadores 
(ou executores), os quais decodificam as ações propostas pelo sistema, para que as mesmas possam ser colocadas em prática. As conseqüências encadeadas a partir de cada ação (retroalimentação do ambiente) determinam a recompensa adequada ao classificador responsável pela ação.

Para formação da população de classificadores, considera-se que cada classificador pode ser tomado como solução, ou uma hipótese para a solução do problema. Observe na Tabela 2 que os classificadores são compostos por uma parte antecedente e outra conseqüente, como usualmente empregado na síntese de regras de inferência.

Associada a cada classificador, temos a sua energia, utilizada para expressar o vigor, ou força, de cada um durante o processo evolutivo.

O casamento da parte antecedente do classificador com a mensagem do ambiente definirá quem irá "competir" ou não. A competição, neste caso, caracteriza-se por uma forma de seleção dos classificadores que se identificaram com a mensagem do ambiente, os quais disputarão o direito de atuar sobre o ambiente. No exemplo da tabela 2, os classificadores B e D, caso sejam selecionados, estarão mais aptos a vencer a competição, devido ao grau de energia que possuem.

Outro conceito importante é o da especificidade de cada classificador, medida inversamente proporcional à quantidade de símbolos "\#" (don't care) na parte antecedente do classificador (Tabela 2).

Tabela 2: Exemplo de classificadores

\begin{tabular}{|c|c|c|}
\hline Hipótese & Classificadores ou Regras & Energia \\
\hline $\mathrm{A}$ & $1 \# 1 \# \#: 11$ & 8,5 \\
\hline $\mathrm{B}$ & $1110 \#: 01$ & 15,2 \\
\hline $\mathrm{C}$ & $11111: 11$ & 5,9 \\
\hline $\mathrm{D}$ & $\# \# 0 \# \#: 10$ & 19,0 \\
\hline
\end{tabular}

Por exemplo, os classificadores A e D são menos específicos, podendo, portanto, identificar-se com um maior número de mensagens do ambiente. Suponha que cada bit da mensagem 10100, de tamanho $\mathrm{L}=5$, caracterize um tipo de informação do ambiente. O classificador A se identificaria com a mesma e também com outras 7 mensagens. Entretanto, o classificador $\mathbf{C}$ se identificaria apenas com a mensagem 11111.

A parte conseqüente do classificador é separada pelo símbolo ":" da parte antecedente, e seu valor determina a ação a ser aplicada ao ambiente por intermédio dos

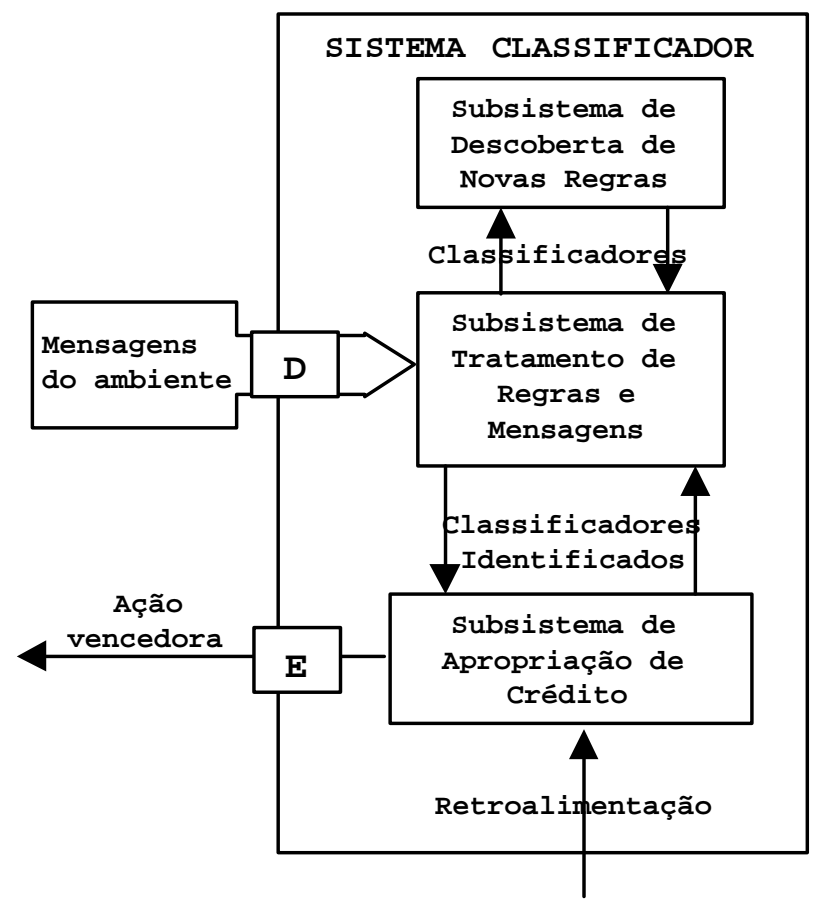

Figura 4: Fluxo simplificado envolvendo o sistema classificador e o ambiente (D - detectores, E - executores)

atuadores. Por exemplo, nos classificadores A e C, a seqüência 11 poderia significar "siga em frente", determinando um dentre 4 movimentos possíveis de um veículo autônomo, em um ambiente a ser explorado.

A parte antecedente do Sistema Classificador é um vetor de tamanho fixo, formado pela concatenação dos caracteres "0", "1" e "\#", os quais são elementos do conjunto $\{0,1, \#\}$. O caractere "\#" conhecido como o símbolo "don't care", pode assumir o valor "1" ou "0", durante a fase de comparação (descrita na seção 4.1). A parte conseqüente é um vetor de tamanho fixo, formado pela concatenação dos caracteres " 0 " e "1" do alfabeto binário $\{0,1\}$, quando se emprega codificação binária, e pela concatenação de números reais, quando se emprega codificação em ponto flutuante.

Internamente, os Sistemas Classificadores dividem-se em três subsistemas distintos, que interagem entre si - Tratamento de Regras e Mensagens, Apropriação de Crédito e Descoberta de Novas Regras. Descreve-se a seguir cada um destes subsistemas, em sua forma básica, existindo a possibilidade de incorporação de mecanismos alternativos àqueles que serão apresentados. 


\subsection{Subsistema de Tratamento de Regras e Mensagens}

Quando os "detectores" de mensagens percebem a presença de alguma mensagem do ambiente, esta é enviada ao Subsistema de Tratamento de Regras e Mensagens (Figura 4). Este codifica a mensagem, de forma que o Sistema Classificador possa reconhecê-la, e coloca a mesma à disposição para a fase de comparação. A partir deste momento, todos os classificadores tentam identificar sua parte antecedente com a mensagem. A identificação pode ser feita por comparação bit a bit, de acordo com regras específicas, ou apenas pelo cálculo de uma variante da Distância de Hamming (Booker, 1985).

No caso de uma comparação explícita, o casamento dos bits da mensagem com os bits diferentes de "\#" ("don't care") do classificador acontece quando os bits estão na mesma posição dentro do vetor de bits. Os indivíduos que possuírem o maior número de posições idênticas às da mensagem serão escolhidos para participarem da competição.

Cada indivíduo (ou classificador) que se identificar com a mensagem será enviado ao Subsistema de Apropriação de Crédito.

\subsection{Subsistema de Apropriação de Crédito}

Durante esta fase, todos os classificadores que se identificaram com a mensagem do ambiente participarão de uma competição, em que o ganhador é definido por um parâmetro chamado "bid", calculado a partir de sua energia e especificidade, modulados por um ruído gaussiano. Ao ganhador será concedido o direito de atuar sobre o ambiente.

Diversas taxas são cobradas de todos os indivíduos da população. A taxa de vida é cobrada de cada classificador a cada iteração. Uma taxa de participação em termos de energia é cobrada de cada indivíduo que participou da competição. O ganhador da competição paga também uma taxa por ter o direito de atuar no ambiente.

O ambiente responderá à ação determinada pelo classificador, fornecendo uma retroalimentação para o Sistema Classificador (Figura 3). É responsabilidade do Subsistema de Apropriação de Crédito incorporar o valor calculado a partir desta retroalimentação à energia do classificador ativo no momento.

Uma vez recebida a retroalimentação do ambiente e atribuído o crédito ao classificador vencedor, uma nova men- sagem será fornecida pelo ambiente, caracterizando o seu novo estado. Volta-se novamente ao tratamento de mensagens e apropriação de crédito. O processo continua por uma época de iterações. Ao final de cada época, o Sistema Classificador participará de outra fase de sua evolução, a fase de descoberta de novas regras.

\subsection{Subsistema de Descoberta de Novas Regras}

Ao final de cada época de iterações são aplicados os procedimentos intrínsecos aos algoritmos genéticos. Neste momento, todas as vantagens desta ferramenta são exploradas em busca de uma população de classificadores cada vez mais adaptada e capaz de atuar em harmonia com o ambiente.

Basicamente, o algoritmo genético escolhe os classificadores com maior energia e promove o cruzamento entre eles, aplicando os operadores genéticos de crossover e mutação. Os descendentes gerados serão inseridos na população obedecendo ao mecanismo de substituição dos indivíduos mais fracos adotado em Richards (1995).

\subsection{Algoritmo Simplificado do Funciona- mento do Sistema Classificador}

De forma simplificada, o algoritmo está representado na figura 5 .

\section{SISTEMAS CLASSIFICADORES APLI- CADOS AO PROBLEMA}

A função do Sistema Classificador é apontar a configuração de chaves ideal para redução das perdas, para cada nova situação de demandas verificadas nos nós que compõem o grafo.

Conforme ilustrado na figura 3, o Sistema Classificador deve comunicar-se com o ambiente através dos "detectores" de mensagens, "executores" e "retroalimentação".

\subsection{Mensagem da Rede de Distribuição}

A rede de distribuição passa como mensagem ao Sistema Classificador o fluxo de potência em cada arco do grafo. Uma vez captada esta mensagem pelos "detectores", ela deve ser codificada de forma a ser processada internamente.

Para simplificar a exposição considera-se apenas os fluxos de potências ativas nas redes (no entanto, a inclusão de fluxos de potências reativas não acrescenta dificulda- 


\section{IIIICIO DO PROCESSO EVOLUTIVO INÍCIO DE UMA ÉPOCA DE ITERAÇÕES}

Passo 1 : Detectar mensagens do ambiente.

Passo 2 : Codificar mensagens.

Passo 3 : Selecionar classificadores que identificam-se com a mensagem.

Passo 4 : Iniciar "Competição" : Calcular "bid" de cada competidor. Apontar vencedor.

Cobrar taxas dos participantes.

Passo 5 : Atuar sobre o ambiente.

Passo 6 : Receber retroalimentação do ambiente.

Passo 7 : Recompensar ou não o classificador vencedor.

Passo 8 : Cobrar taxa de vida de todos os

classificadores.

Passo 9 : Caso não seja fim de uma "Época", retornar ao Passo 1.

\section{FIM DE UMA ÉPOCA DE ITERAÇÕES}

Passo 10 : Selecionar classificadores para aplicação do algoritmo genético.

Passo 11 : Aplicar os operadores de crossover e mutação para gerar os filhos.

Passo 12 : Selecionar os indivíduos mais fracos da população.

Passo 13 : Inserir os filhos gerados no Passo 11 na população, substituindo os indivíduos selecionados no

Passo 12.

Passo 14 : Caso não seja fim do processo evolutivo, voltar ao Passo 1.

FIM DO PROCESSO EVOLUTIVO

Figura 5: Algoritmo Simplificado

Tabela 3: Quantização dos fluxos de potência

\begin{tabular}{|c|c|c|c|}
\hline & $\begin{array}{r}\text { Fluxc } \\
\text { Cada S }\end{array}$ & $\begin{array}{l}\text { SETORES } \\
0 \text { a } f \max (\mathrm{Kw}) \\
\text { tor : } f \max / 4(\mathrm{Kw}\end{array}$ & \\
\hline $\begin{array}{c}1 \\
\mathrm{x}_{0}=0\end{array}$ & $\begin{array}{c}2 \\
\mathrm{x}_{1}=\mathrm{x}_{0}+f \max / 4\end{array}$ & $\mathrm{x}_{2}=\mathrm{x}_{1}+f \max / 4$ & $\begin{array}{c}4 \\
\mathrm{x}_{3}=\mathrm{x}_{2}+f \max / 4\end{array}$ \\
\hline
\end{tabular}

des conceituais). Para cada configuração de rede, os fluxos são calculados recursivamente a partir dos extremos dos alimentadores até as subestações. Assim, não há "inversão de fluxos" - em outras palavras, os fluxos são sempre positivos.

O fluxo de cada arco, em ponto flutuante, é quantizado para transformar-se em uma representação de 2 (dois) bits. As tabelas 3 e 4 ilustram o processo de codificação da mensagem. Os fluxos são classificados em quatro setores. Suponhamos que temos um fluxo máximo fmax. O setor 1 engloba os fluxos $0 \leq f<(f \max / 4)$; o setor 2 , os fluxos de $(\max / 4) \leq f<(f \max / 2)$; e assim por diante (veja Tabela 3 ).

Tabela 4: Exemplo de codificação $(\operatorname{fmax}=3140)$

\begin{tabular}{|c|c|c|c|}
\hline SETOR & DE & ATÉ & EM BINÁRIO \\
\hline 1 & 0 & 785 & 00 \\
\hline 2 & 785 & 1570 & 01 \\
\hline 3 & 1570 & 2355 & 10 \\
\hline 4 & 2355 & 3140 & 11 \\
\hline
\end{tabular}

Identificado o setor ao qual cada fluxo $f$ pertence, este é transformado em uma seqüência binária. Continuando o exemplo anterior, seja $f \max =3140$ e considere $f_{1}=$ 1680 como o fluxo no arco 1 . Neste caso $f_{1}$ pertence ao setor 3 e é representado pela seqüência " 10 ". Portanto, adotando-se esta codificação, o número de bits (0's e 1's) da mensagem da rede de distribuição vai corresponder a duas vezes o número de arcos do grafo.

\subsection{Parte Antecedente dos Classificadores}

A parte antecedente do Sistema Classificador é um vetor de tamanho fixo, formado pela concatenação dos caracteres "0", "1" e "\#", os quais são elementos do conjunto $\{0,1, \#\}$. Neste caso o tamanho desse vetor corresponde ao tamanho da mensagem da rede, ou seja, duas vezes o número de arcos.

A parte antecedente do classificador deverá identificar-se com a mensagem do ambiente. Observe que a presença do caractere "\#" na parte antecedente associa uma certa capacidade de generalização ao antecedente do classificador.

\subsection{Parte Conseqüente dos Classificadores}

A parte conseqüente de cada classificador é representada com dois níveis de codificação (Costa, 1999): codificação direta e codificação indireta. Na codificação indireta a parte conseqüente é composta por um vetor de pesos fictícios (valores em ponto flutuante) no intervalo fechado $[0,1]$, atribuídos a cada arco do grafo que representa a rede de distribuição. Aplicando o algoritmo gerador de árvores de custo mínimo de Sollin (Ahuja et al., 1993) a este vetor, conseguimos gerar uma configuração radial para a rede de distribuição, especificando assim as chaves abertas e as chaves fechadas (codificação direta).

\subsection{Caracterização de Parâmetros}

Entendem-se como dados da rede as especificações consideradas como invariantes nesta abordagem do problema (número de nós de demanda, número de arcos, ligações 
entre os nós, resistências, reatâncias, demandas máximas, tensão no barramento da subestação). A população inicial de indivíduos (classificadores ou regras) é criada aleatoriamente - no estudo de casos considerouse uma população de 500 indivíduos. Na parte antecedente, a probabilidade inicial de encontrar-se o símbolo "\#" é de 70\%.

É criado um conjunto de treinamento com configurações de demandas diferentes, que requerem configurações de chaves distintas, as quais são desconhecidas, correspondendo justamente à saída que o Sistema Classificador deve produzir.

Cada "época" compreende 150 iterações, com um número máximo de 30 épocas (dependendo do conjunto de treinamento). Calcula-se a recompensa a partir da retroalimentação do novo estado do sistema, para cada configuração proposta pelo classificador vencedor.

Em princípio, determina-se um número máximo de 4.500 iterações por perfil de demanda apresentado. O algoritmo genético é aplicado a $10 \%$ da população de classificadores selecionada, baseando-se na energia de cada um (fitness). A percentagem de crossover é de $100 \%$ e a de mutação $1 \%$.

São criados parâmetros distintos para aplicação dos operadores a cada parte do indivíduo (antecedente e conseqüente). Na parte antecedente, são aplicados o crossover simples e mutação com $50 \%$ de probabilidade para cada "alelo" (bit) restante. Na parte conseqüente, são aplicados crossover simples e mutação específica para representações em ponto flutuante (Michalewicz, 1992).

\section{ESTUDO DE CASOS}

Caso 1: Perfil de demanda fixo: solução usando Sistemas Classificadores

Embora se tenha consciência de que Sistemas Classificadores são particularmente adequados para fornecer soluções no caso de "ambientes" variantes no tempo, vamos inicialmente verificar a capacidade de convergência do Sistema Classificador para perfis de demanda fixos.

Empregou-se o Sistema Classificador descrito na seção anterior ao perfil P1, previamente utilizado na aplicação de algoritmos genéticos (Seção 3.1). O melhor resultado encontrado pelos sistemas classificadores indicou a seguinte configuração de chaves abertas: 7, 10, 14, 16 e 27 . Esta configuração é muito próxima do melhor resultado encontrado pelo algoritmo genético (chaves abertas: 7 , $10,14,16$ e 28$)$.
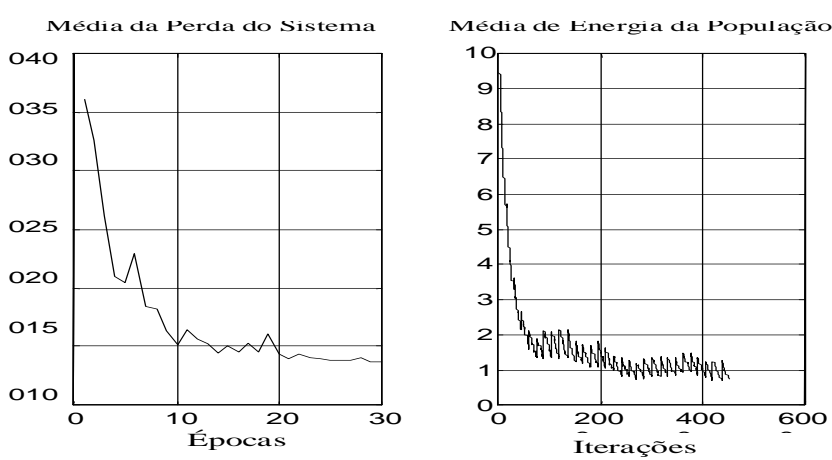

Figura 6: Desempenho e Evolução do Sistema Classificador

Na figura 6, gráfico à esquerda, é apresentada a média de desempenho do sistema na redução das perdas. $\mathrm{Na}$ figura 6 , gráfico à direita, temos a evolução da média de energia da população de classificadores.

Caso 2: Perfil de demanda variável: solução usando Sistemas Classificadores

Os resultados obtidos no Caso 1 e ilustrados na figura 6 são promissores no sentido de indicar que o Sistema Classificador é capaz de partir de um conjunto de soluções de baixa qualidade (condição inicial do Caso 1) e convergir para uma boa solução, em um tempo adequado. O Caso 2 estuda situações em que o perfil de demanda do sistema sofre variações abruptas (em degrau), representando praticamente o reinício do processo de geração de classificadores.

Escolhemos dois perfis, 1 e 4, com demandas distintas. Iniciamos o processo evolutivo com o primeiro perfil e fizemos a transição gradativa a cada 20 épocas para o segundo perfil, escolhendo dois pontos intermediários, perfis 2 e 3 , os quais caracterizam pontos de transição.

Adotamos, como parâmetro para análise de desempenho, a média da perda de energia apresentada pelo sistema de distribuição durante a evolução do Sistema Classificador.

Resultados obtidos (em p.u.) com os quatro perfis de demanda, ilustrados na figura 7 , indicam que o Sistema Classificador desenvolvido é capaz de evoluir de um conjunto de soluções inadequadas (condição inicial) para boas soluções.

Acompanhando a seqüência dos perfis introduzidos, mostramos a seguir, as configurações propostas pelo Sistema Classificador: 


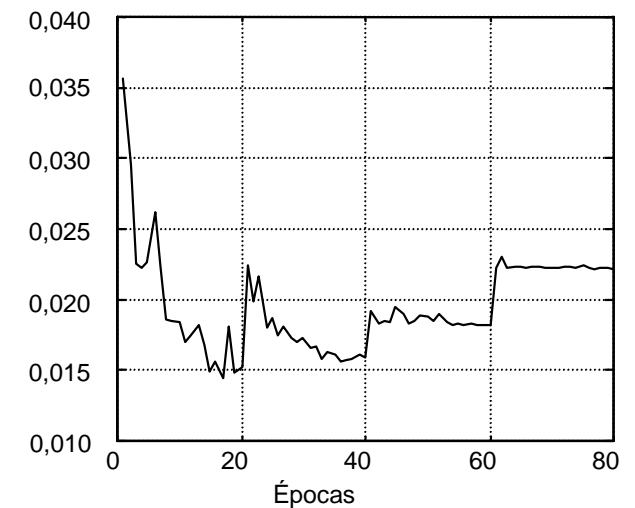

Figura 7: Desempenho do Sistema Classificador

- PERFIL 1: $10 / 15 / 25 / 33 / 34$

- PERFIL $2: 7 / 10 / 15 / 27 / 34$

- PERFIL $3: 7 / 10 / 12 / 16 / 28$

- PERFIL 4 : $7 / 9 / 12 / 16 / 28$

O Sistema Classificador foi suficientemente flexível para indicar novas soluções sempre que as variações de demanda começavam a interferir significativamente no nível de perdas associado a uma determinada configuração da rede de distribuição (estado das chaves).

Finalmente na última etapa do Caso 2 (Figura 8), submetemos o Sistema Classificador a variações suaves e cíclicas de demanda, introduzindo a cada 15 épocas os perfis da etapa anterior na seguinte ordem: $1,2,3,4,3$, $2,1,2,3$ e 4 .

Podemos observar na figura 8 o desempenho quase ótimo do Sistema Classificador, confirmando suas características. A cada mudança periódica e suave de perfil, o sistema foi capaz de não somente se adaptar à nova situação do ambiente como também melhorou seu desempenho a cada nova mudança, chegando nas últimas simulações quase que a uma condição estável. Este fato retrata um comportamento esperado de um sistema complexo adaptativo, onde a reapresentação de um dado estado do sistema produz uma resposta de melhor qualidade. É muito provável que esta característica esteja associada ao fato de que as regras que venceram na última vez que o perfil foi apresentado ainda estivessem presentes na população de regras.

Portanto podemos afirmar que o Sistema Classificador é um sistema suficientemente flexível para adaptar-se a novos contextos, nunca antes apresentados, e também eficaz na produção de respostas secundárias à reapresentação de estímulos do ambiente.

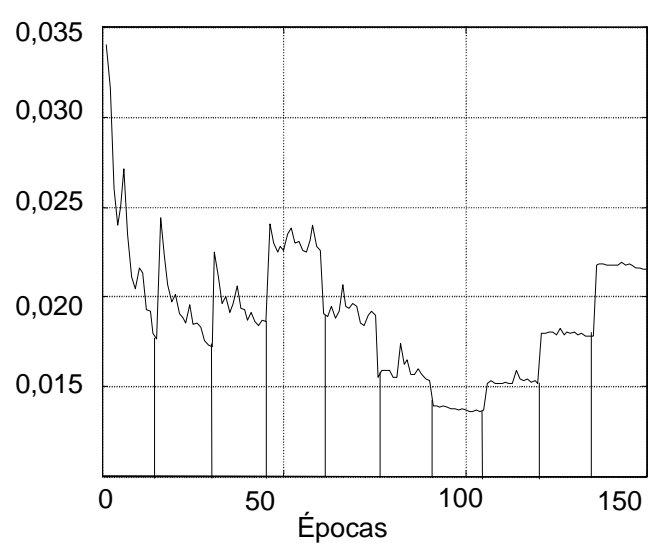

Figura 8: Desempenho do Sistema Classificador

\section{CONCLUSÃO}

O trabalho apresentou os desenvolvimentos metodológicos necessários à aplicação de Sistemas Classificadores na minimização de perdas técnicas em sistemas de distribuição de energia elétrica.

Os Sistemas Classificadores mostraram-se adequados a uma atuação adaptativa, em situações com variações de demanda nos barramentos.

Embora dependam da definição de um conjunto de parâmetros para o sucesso das implementações, os estudos de casos indicaram que os Sistemas Classificadores constituem uma abordagem promissora para esta classe de problemas.

Para situações de demandas fixas, observamos que os algoritmos genéticos puros obtiveram configurações de redes com características de perdas ligeiramente melhores. Isto ocorre em função dos algoritmos genéticos permitirem uma melhor exploração da característica estacionária do ambiente, ao passo que os Sistemas Classificadores precisam manter a flexibilidade para adaptação a novas situações; se o ambiente permanece invariante, esta flexibilidade é de pouca utilidade.

Por outro lado, em situações de demandas variáveis, os Sistemas Classificadores reagiram com agilidade às mudanças do ambiente, propondo configurações distintas, adequadas a cada novo perfil de demanda apresentado. Com algoritmos genéticos puros, a população que levou a uma boa característica de perdas para um determinado perfil de demanda não permitia a obtenção de uma boa configuração quando a demanda sofria alteração. Isto é conseqüência da perda de diversidade, cuja reversão pode ser interpretada como o reinício do pro- 
cesso evolutivo, sem a possibilidade de aproveitamento das adaptações fornecidas até então.

Vale relembrarmos que o principal benefício da aplicação de sistemas classificadores ao problema abordado neste trabalho é a possibilidade de aplicação à supervisão em tempo real de redes de distribuição, propondo configurações que levem à redução de perdas sempre que forem identificadas variações significativas de demanda.

Este trabalho trouxe uma contribuição inicial para avaliação dessas possibilidades. No entanto, deixa ainda muitos aspectos a serem considerados. Entre esses, o estudo de outras arquiteturas de sistemas classificadores e avaliações de aspectos técnicos associados a reconfigurações, como desgaste das chaves seccionadoras e impacto nos sistemas de proteção.

\section{AGRADECIMENTOS}

Este trabalho teve apoio do CNPq, CAPES e FAPESP. Os autores agradecem também ao colega Celso Cavellucci, pela colaboração e sugestões, e aos revisores anônimos, pelos comentários e sugestões.

\section{REFERÊNCIAS}

Ahuja, R. K., Magnanti, T. L. \& Orlin, J. B. (1993). Network Flows: Theory, Algorithms and Applications. Prentice Hall, Englewood Cliffs, NJ.

Booker, L. B. (1985). Improving the Performance of Genetic Algorithms in Classifier Systems. Proc. of an Int. Conf. on Genetic Algorithms and their Applications, pp. 80-92.

Booker, L. B., Goldberg, D. E. \& Holland, J. H. (1989). Classifier Systems and Genetic Algorithms. Artificial Intelligence, vol. 40, pp. 235-282.

Cavellucci, C. \& Lyra, C. (1997). Minimization of Energy Losses in Electric Power Distribution Systems by Intelligent Search Strategies. International Transactions in Operational Research, vol. 4, no. 1, pp. 23-33.

Civanlar, S., Grainger, J. J., Yin, H. \& Lee, S. S. H. (1988). Distribution feeder Reconfiguration for Loss Reduction. IEEE Transactions on Power Delivery, vol. 3, pp. 1217-1223.

Costa, M. F. N. (1999). Computação Evolutiva para Minimização de Perdas Resistivas em Sistemas de Distribuição de Energia Elétrica. Tese de Mestrado, FEEC, Unicamp, Julho.
Glover, F., Laguna, M. (1997). Tabu Search. Kluwer Academic Publishers.

Holland, J. H. (1992). Adaptation in Natural and Artificial Systems: an Introductory Analysis with Applications to Biology, Control, and Artificial Intelligence. The MIT Press, Ann Arbor, MI.91.

Lanzi, P. L., Stolzmann, W. and Wilson, S. W., editors (2000). Learning Classifier Systems. From Foundations to Applications, volume 1813 of LNAI. Springer-Verlag, Berlin.

Lanzi, P. L., Stolzmann, W. and Wilson, S. W., editors (2001). Advances in Learning Classifier Systems, volume 1996 of LNAI. Springer-Verlag, Berlin.

Merlin, A. \& Back, H. (1975). Search for a Minimal-Loss Operating Spanning Tree Configuration in Urban Power Distribution Systems. Proc. of 5 th Power Systems Comp. Com., Cambridge, U.K., Sept. 1-5.

Michalewicz, Z. (1996). Genetic Algorithms + Data Structures = Evolution Programs.. Springer-Verlag Berlin Heidelberg, New York.

Nara, K. A., Shiose, M., Kitagawa \& Ishihara,T. (1992). Implementation of Genetic Algorithm for Distribution Systems Loss Minimum Reconfiguration. IEEE Transactions on Power Systems, vol.7,no.3, pp.1044-1051.

Peponis, G. J., Papadopoulos, M. P. \& Hatziargyriou, N. D. (1995). Distribution Network Reconfiguration to Minimize Resistive Line Losses. IEEE Transactions on Power Delivery, vol. 10, no. 3, pp. 1338-1342.

Richards, R. A. (1995). Zeroth-Order Shape Optimization utilizing Learning Classifier Systems. PhD. Dissertation, University of Stanford. URL: http://www.stanford.edu/ buc/SPHINcsX/book.html

Shirmohammadi, D. \& Hong, H. W. (1989). Reconfiguration of electric distribution networks for resistive line losses reduction. IEEE Transactions on Power Delivery, vol. 4, pp. 1492-1498.

Vargas, P.A., Lyra Filho, C. \& Von Zuben, F.J. (2001) Classifier Systems for Loss Reduction in Electric Power Distribution Networks. Proceedings of the Fourth International Workshop on Learning Classifier Systems (IWLCS-2001), GECCO-2001, pp. 372-376, San Francisco, California, USA, July 7-8.

Vargas, P. A., de Castro, L. N. \& Von Zuben, F. J. (2003). "Mapping Artificial Immune Systems into Learning Classifier Systems", In Lecture Notes in Artificial Intelligence (LNAI) Springer-Verlag (in print). 